\title{
Cell-based assays in high-throughput mode (HTS)
}

\author{
KALYANI RAJALINGHAM \\ Universite du Quebec a Montreal, Department of Biological Sciences, Montreal, Canada
}

\begin{abstract}
Typically, novel compounds are screened by the millions, a process known as high-throughput screening (HTS). HTS allows for the screening of millions of potential drugs in a relatively short period of time. All compounds are initially subjected to various tests to determine safety and efficacy. At the molecular level, typically two types of tests are available: in vitro and cell-based assays (i.e., in vivo). The distinction between a cell-based assay and an in vitro screening is that the cell-based assay utilizes live cells - approximately 50000 cells are seeded onto the floor of the well. Cell-based assays are used to measure proliferation, toxicity, marker production, motility, activation of signaling pathways, and changes in morphology. In such cases, other factors such as 2D versus 3D culture or static versus profusion cultures might also contribute to the results obtained. This study emphasizes the positive aspects of using cell-based assays in high-throughput mode.
\end{abstract}

Key words: cell-based assay, high-throughput screening, drug discovery

\section{Introduction}

At least $50 \%$ of drugs available on the market target G-coupled protein receptors (GPCRs) (Chattopadhyay, 2014; Tautermann, 2014; Takakura et al., 2015), making this protein family the most pharmacologically significant. A number of methods have been developed and tailored to assay GPCR activity. Activation of a GPCR by its ligand results in receptor activation, receptor internalization/recycling, activation of second messenger, cytoskeletal remodeling, expression of target genes, and cell adherence among others (Lodish et al., 2000; Fang et al., 2008). Assays aiming at GPCRs should be nonradioactive and homogenous, and should not utilize much reagents (due to cost) (Thomsen et al., 2005). Radioactive assays - assays that use radioactive ligands - are not homogeneous and requires many washes which implies that it is both complex and expensive (Cottet et al., 2011). Radioactive ligands have to be properly disposed as they can constitute a health hazard and require safety precautions in the work area (Cottet et al., 2011). There are mainly two types of assays that can be performed in high-throughput screening (HTS) mode: the biochemical assay and the cell-based assay. Although biochemical assays have their advantages, many compounds cannot be extracted in quantity and purity suitable for biochemical assays; furthermore, biochemical assays, while allowing precise characterization of molecular interactions, may not be representative of the actual effect in a cellular milieu where multiple biochemical pathways function simultaneously and may compete, synergize, and/or compensate for each other (Zang et al., 2012). In such cases, cell-based assays - using live cells - are often required to determine the effect of a target compound on a cell. Cell-based assays can be used to assess proliferation, toxicity, production of markers, motility, activation of specific signaling pathways, and changes in cell morphology (Yeon and Park, 2005). This study reviews the positive aspects of cell-based assays in high-throughput mode.

\section{Cell-based assays in HTS mode}

Typically, animals are required to copiously simulate in vivo effects, however, cell-based assays can be classified as semi-in vivo in that they use live cells and that they allow the investigator to identify molecules with desired activities and those devoid of unwanted characte- 
ristics such as cell toxicity and low cell uptake. Also, HTS cell-based assays minimize the number of animals sacrificed to experimentation, costs (cryopreserved mice, for instance, costs $\$ 2525.00$ USD and have a high maintenance cost in specialized and expensive facilities), and time (to raise, feed, etc.) (Breier et al., 2008; Macarron et al., 2011; Szymański et al., 2011). HTS cell-based assays allow the testing of millions of compounds efficiently and rapidly prior to animal testing; testing on animals is then performed only on promising (lead) candidate compounds. In fact, high throughput implies the testing of up to 100000 compounds per day, and with the latest ultra-high throughput techniques, outputs can exceed those numbers (Szymański et al., 2011). The use of HTS yielded approximately 120 leads (potential drugs) per year per laboratory, resulting - just in the year 2004 - in 104 drug candidates progressing to clinical trials from the collective work of 26 labs (Fox et al., 2006). However, over $60 \%$ of the drugs that failed during clinical trials were also HTS based (Macarron et al., 2011; Zhang et al., 2012), and therefore, there is a need to develop more efficient ways to screen compounds that can provide high confidence lead candidates.

Cell-based assays are carried out in either 96- or 384well plates with approximately 10000 cells per well (Breier et al., 2008; Peterson et al., 2014). Volumes of $25-50 \mu$ reagent per well for a 384 well plate is standard (Frank An and Tolliday, 2010) allowing for reagent economy, which is especially important when using expensive or limiting reagents (e.g., antibodies). Moreover, three types of detection systems are frequently used: colorimetric, fluorescent, and luminescent detection, all of which are easily carried out in a fully automated mode. However, these detection systems are not without flaws. The luminescent system requires an endpoint assay. An endpoint assay is one that can only be carried out at the end of a reaction; the luminescent system requires the addition of multiple compounds (such as luciferase and luciferin) that typically requires the cells to be sacrificed prior to the addition of the compounds. The use of a luciferase from Metridia longa, instead of firefly, would permit a live cell assay without the need for lysis; however, factors such as $\mathrm{pH}$, and buffer can affect the signal and must be optimized (Zang et al., 2012). Further, the luminescent system also has a low background luminescence, thereby producing a strong stable signal for a few minutes and is commercially acquirable (Gibbs, 2001). The fluorescent system is the most commonly used detection system and includes techniques such as fluorescence intensity, fluorescence polarization, fluorescence resonance energy transfer, fluorescence lifetime, and time-resolved fluorescence (Frank An and Tolliday, 2010). The fluorescent detection system permits real-time analysis, and does not require staining, fixation, and permeabilization steps which are normally required for alternate techniques (Kain, 1999). However, common problems associated with fluorescence-based assays are quenching, scattering of signal, and intrinsic autofluorescence of some tissues (Rogers, 1997). The colorimetric assay typically utilizes ruthenium dye and Alamar Blue; however, the sensitivity of ruthenium dye and reliability of Alamar Blue are poor which intimates that they are not appropriate for HTS (Zang et al., 2012). Alternatively, one can resort to using tetrazolium salts (MTT, MTS, and XTT) which when reduced produce a colored product. MTT assays are used to assess viability of cells, cell proliferation, and mitochondrial activity by quantifying the amount of DNA present in a well of the microtiter plate (Papadimitriou and Lelkes, 1993). The MTT preparation is subsequently added to the cells; and the conversion of MTT to formazon by the mitochondria is indicative of cell viability. Given that formazan is purple in color and has an absorbance of $570 \mathrm{~nm}$, optical density measurements - using a spectrophotometer - can be used for detection. Unviable cells are incapable of converting MTT to formazon. MTT is frequently used in the assessment of proliferation of retinal pigment epithelium (Yu et al., 2003). However, MTT assays are deemed robot unfriendly and are nonhomogeneous; they require pre-incubation and washing steps (Slater, 2001). Furthermore, MTT results in the formation of crystals which must be solubilized before visualization; since the solubilization process itself is rather hard, well-to-well differences might occur. The MTS (3-(4,5-dimethylthiazol-2-yl)-5-(3-carboxymethoxyphenyl)-2-(4-sulfophenyl)-2H-tetrazolium) or the XTT (2,3-bis-(2-methoxy-4-nitro-5-sulfophenyl)-2H-tetrazolium-5-carboxanilide) assays are used as an alternative to the MTT assay. XTT is cleaved by viable cells to form aqueous formazan (which is already solubilized) (Riss et al., 2013); MTS, in the presence of NADH and $\mathrm{NADPH}$, reduces to water-soluble formazan. However, use of the XTT method does not allow for additional procedures such as flow cytometric analysis on the XTT- 
treated cells (Yu et al., 2003). Tetrazolium salts (WSTs) are also used instead of MTT; WSTs results in the production of water-soluble formazan and not crystals. However, the disadvantage of such a method is that it is tedious, is dilatory, is an endpoint assay, and it necessitates the addition of compounds that can damage the cells (Zang et al., 2012).

Moreover, cell density, reagent, modulator concentration, and incubation time must be optimized; reagent and signal stability must be determined, as well as the possible effect of the solvent alone (Frank An and Tolliday, 2010). Care must also be taken in the selection of plates. For instance, solid black polystyrene microplates can minimize cross-talk between wells and fluorescence when using a fluorescent detection system; meanwhile, solid white polystyrene is used in conjunction with luminescent detection systems (Zhang et al., 2012; Corning Life Sciences, 2015). Variation between wells, plates, day, and batch should be assessed as well. Primary assays are utilized to detect hits; and secondary assays are utilized to confirm said hits (Johnston and Johnston, 2002). All hits must be subject to in vivo testing prior to clinical trials, and cellbased assays allow for rapid initial testing.

The process of drug screening involves four phases: early and late discovery, preclinical and clinical phases; the two main reasons for rejection are the efficacy of the drug and its toxicity (McKim, 2010). The majority of hits rejected at this stage are due to cell toxicity. Identification of a potential drug is followed by optimization and pre-clinical animal toxicity studies (Schoonen et al., 2009). Testing for toxicity is usually carried out before the phase I clinical stage (Slater, 2001). Due to their toxic effects on the cells, approximately $40-50 \%$ of drugs do not reach the market (Schoonen et al., 2009). It was also reported that about $3 \%$ of the drugs that made it to the market were then recalled due to adverse side effects (liver, kidney, gastric, and cardiac toxicity); another $10 \%$ were placed on the "black box" warning list by the FDA (Astashkina et al., 2012) as drug toxicity can result in organ or tissue damage. Toxicity screens are carried out by testing for pre-lethal indicators. Toxic effects of a drug on a cell usually result in a signaling cascade that causes a change in the polarization of cells, alteration of gene expression, and post-translational modifications, or permeability (Astashkina et al., 2012). To date, toxicity screens are only carried out on known pathways, and as such, if the pathway of the drug is unknown, toxicity is left undetected and usually picked up during pre-clinical or clinical studies. Further, most toxicity tests assess short-term and not the long-term effects. There are many toxicity assays; the following are standard assays: endocrine disruption assays, nuclear receptors and CYP 450 enzyme induction, CYP 450 enzyme competition assays, embryotoxicity assays, clastogenicity assays, cytotoxicity assays, mutagenicity assays, and non-genotoxic carcinogenicity assays. In fact, $40 \%$ of the lead compounds that enter the preclinical phase and $89 \%$ of the lead compounds that enter the clinical phase fail due to toxicity; and only about 1 in 10,000 leads are marketed (McKim, 2010). Since toxicity is multi-faced - it can occur at various location/tissue or time. A number of screens are required to determine effects. The ratio of toxicity (TC50) to efficacy (IC50) is used to compare multiple compounds (McKim, 2010).

Cell-based assays begin with the selection and production of an appropriate cell line (primary or engineered, e.g., addition of a reporter gene), followed by compound exposure and signal capture (Johnston and Johnston, 2002; Zhang et al., 2012). In particular, GPCRs often possess post-translational modifications, therefore, a cell line that also possesses such modifications must be selected (Thomsen et al., 2005). GPCRs are typically modified post-transcriptionally and they are ofttimes associated with GIPs (G-protein-interacting proteins); they can activate multiple G-proteins, and at times form homodimers or heterodimers (with similar or different GPCRs); as such, the selected cell line must incorporate all factors (Thomsen et al., 2005). If the chosen cell line does not reproduce these multiplicity factors, then many potential drugs are likely be missed. Furthermore, one must decide on which components of the affected pathways are to be measured - proximal (e.g., cAMP levels, $\mathrm{Ca}^{++}$release) or distal targets (e.g., target gene activation). Measurement of proximal components lowers false positives, while the signal-to-noise ratio $(\mathrm{S} / \mathrm{N})$ is usually higher when distal targets are measured (Thomsen et al., 2005). Further, cytotoxicity due to exposure to a compound can lead to false positives or false negatives (Johnston and Johnston, 2002).

In HTS mode, the aim is to maximize speed, efficiency, sensitivity, reproducibility, accuracy, and signal detection, while minimizing use of reagents and error (Zhang et al., 1999). A hit is recorded when the signal emitted by the addition of a compound is above a thre- 
shold value of the sample average. However, one must note both the signal-to-noise ratio $(\mathrm{S} / \mathrm{N})$ and the signalto-background $(\mathrm{S} / \mathrm{B})$ ratio. The $\mathrm{S} / \mathrm{B}$ ratio is not used since it does not incorporate variability into the equation; as such, the S/B ratio is typically not utilized when assessing the validity of a dataset. The $\mathrm{S} / \mathrm{N}$ ratio is indicative of assay quality; it indicates whether a hit is associated with a true signal. However, the $\mathrm{S} / \mathrm{N}$ ratio does not account for all existing variabilities in a screen. False positives and false negatives can be sieved out by decreasing the variability observed in a data set by using statistical calculations (Zhang et al., 1999).

$$
\frac{S}{N}=\frac{\mu_{\text {signal }}-\mu_{\text {background }}}{\sigma_{\text {background }}}
$$

A better measure for the $\mathrm{S} / \mathrm{N}$ parameter is $Z^{\prime}$ which incorporates experimental variability into the equation. Statistically speaking, within \pm 3 standard deviations, one can observe about $99.73 \%$ of the data. A hit compound would have a signal window such that (Takakura et al., 2015; Zhang et al., 1999):

$$
\begin{gathered}
\left|\mu_{s}-\mu_{c}\right|-3\left(\sigma_{c}+\sigma_{s}\right) \\
Z^{\prime}=\frac{\left|\mu_{s}-\mu_{c}\right|-3\left(\sigma_{c}+\sigma_{s}\right)}{\left|\mu_{s}-\mu_{c}\right|} \\
Z^{\prime}=1-\frac{3\left(\sigma_{s}-\sigma_{c}\right)}{\left|\mu_{s}-\mu_{c}\right|}
\end{gathered}
$$

$s$ - sample, $c$ - control; where $\sigma_{s}, \sigma_{c}$ are standard deviations and $\mu_{s}, \mu_{c}$ are means.

Further, assuming that under perfect conditions variation is small and that the mean of the sample is substantially larger than that of the control, one could further claim that $3\left(\sigma_{c}+\sigma_{s}\right)$ should approach zero and that $\left|\mu_{s}-\mu_{c}\right|$ would be a non-negative large number, then under ideal conditions $Z^{\prime}$ must approach 1 . Theoretically, $Z^{\prime}$ is such that $-\infty<Z^{\prime} \leq 1$; for assays, $Z^{\prime}$ is bounded between -1 and 1 (Zhang et al., 1999) and is used to determine the validity of collected data. In fact, $Z^{\prime}<0$ is considered unfit, while values such that $0<Z^{\prime}<0.5$ are deemed insignificant. On the other hand, $Z^{\prime}>0.5$ implies valid results (Bray and Carpenter, 2013; Frank An and Tolliday, 2010).

\section{$2 \mathrm{D}$ versus $3 \mathrm{D}$ cultures}

To simulate the actual cellular microcosms including cell-cell or cell-matrix interactions, 3D cultures were ge- nerated (Justice et al., 2009). 3D cultures are formed by many methods: the 3D spheroids using a scaffold (an acellular or liquid matrix) or the scaffold-free 3D spheroids method (forced floating method, hanging drop method, or agitation-based approaches) (Fennema et al., 2013; Edmondson et al., 2014). For instance, in the hanging drop method, a pipette is used to dispense the cells through a narrow opening of the hanging drop plate where the cells aggregate into a spheroid (Zang et al., 2012). 3D cultures show higher similarity to the actual primary cells (i.e., from animal tissues), and behavior of cells differ when in $2 \mathrm{D}$ versus $3 \mathrm{D}$ cultures; for instance, proliferation rates differ between $2 \mathrm{D}$ and $3 \mathrm{D}$ cultures. Response to a single drug target was also found to differ between 2D and 3D cultures. Smitskamp-Wilms et al. (1998) conducted an experiment using the human ovarian cancer cell line A2780 to examine the effects of gemcitabine $(\mathrm{dFdC})$ in $2 \mathrm{D}$ (monolayer) and $3 \mathrm{D}$ (multilayer) cultures. Retention of gemcitabine, resulting in cellular growth inhibition, was found to be much higher in $2 \mathrm{D}$ cultures than $3 \mathrm{D}$ cultures (Smitskamp-Wilms et al. 1998). Translated, the latter compound would be deemed effective when tested in 2D cultures and ineffective in 3D cultures. Tung et al. (2011) tested the effect of a known anticancer drug, 5-fluorouracil (5-FU), and a DNA damaging agent, TPZ, on A431.H9 cells under 2D and $3 \mathrm{D}$ culture conditions. The results showed 5-FU to be more effective under 2D culture conditions, which the authors attribute to the effective permeation of drug (Tung et al., 2011). Tung et al. (2011) showed that approximately $75 \%$ of 5 -FU-treated cells under $3 \mathrm{D}$ culture conditions and $5 \%$ of 5 -FU-treated cells under $2 \mathrm{D}$ culture conditions were viable. Furthermore, $40 \%$ of TPZ-treated cells under 3D culture conditions and 70\% of TPZ-treated cells under 2D culture conditions were found to be viable. This would imply that compounds chosen under $2 \mathrm{D}$ culture conditions and rejected under 3D culture conditions may not perform well in clinical trials.

\section{Profusion versus static cultures}

Static cultures are controls for cell-cell and cellmatrix interactions, while microfluidic perfusion cultures serve as control for phenotypes (Kim et al., 2007). In other words, static cultures offer one medium per batch (e.g., 96-well plate). Unlike static cultures, perfusion cultures can be used for experiments that must be 
conducted over a longer period of time without fear of contamination. Further, as opposed to a static culture, a perfusion culture, otherwise known as a microfluidic cell culture, is designed in such a manner as to permit a constant environment within the well. Microfluidic perfusion cultures are used to supply the cellular mass in a well with soluble molecules such as oxygen and nutrients as well as to remove waste products using microfluidic valves, mixers, and generators of gradients (Kim et al., 2007). Microfluidic screening can occur in three modes: perfusion flow mode, droplet-based mode (uses water-in-oil droplets to hinder cross-contamination), and microarray mode (Du et al., 2016). Microfluidic cultures are utilized to mimic the subcellular environment. For instance, kidney and liver cells are in close proximity to the vascular system, and as such, when attempting to assess the effect of a drug on liver or kidney cells, microfluidic cultures are helpful in re-creating a microenvironment that is similar to the in vivo microenvironment. The process itself is divided into three temporally divided sections: cell seeding (channels used to deliver cells at low velocity - to permit settling of cells), perfusion cell culture (circulating or non-circulating culture medium delivered to cells), and cell testing (reagents required for assay delivered to cells) (Kim et al., 2007).

\section{Proliferation}

Cell proliferation is typically assessed by measuring electrochemical impedance, a method known as bioimpedance. Bioimpedance does not require a detection system (colorimetric, fluorescent, or luminescent) or chimeric proteins (Mcguinness, 2007). The base of the well is equipped with electrodes, an alternating voltage is applied, and the electrical current is determined (Mcguinness 2007). The underlying assumption is that impedance is correlated to the number of cells in a well. Bioimpedance (or electrical biosensors) has been commonly used to study cell adhesion, spreading, cell micromotion, and morphological changes as well as the shortterm effects of receptor activation and modifications in cell shape/volume (Mcguinness, 2007; Fang et al., 2008). However, high variability is often observed between assays when using bioimpedance (Fang et al., 2008). Fang et al. (2008) noted that most techniques used in HTS require cellular engineering that might lead to modifications in the potency of the drug. In other words, one would be measuring the efficacy of the drug on the engineered counterpart of the cell, and therefore, optimizing selection of drug to the engineered counterpart, which may not faithfully reproduce the normal cell. In such cases, non-engineered cell lines, systems, and techniques are valuable to assess, select, and optimize drugs. Proliferation can also be assessed by gauging the amount of DNA present in the well. For instance, Bedard et al. (1999) used a human fibroblast (Hs68) cell line and a colorimetric proliferation assay using WST-1. WST-1 is a tetrazolium salt that can be turned into a colored product - formazan - that can be detected and used to determine cell number. Results were found to vary with seeding cell density, drug load, and incubation period (Bedard et al., 1999). In this case, the authors noted the effect of $2 \mathrm{D}$ versus $3 \mathrm{D}$ cultures on the efficacy of the drug.

\section{Changes in cell morphology}

Since activation of a GPCR receptor ultimately leads to phenotypic changes in the cell (Fang et al., 2008), bioimpedance can be used to assess changes in cell morphology. Receptor binding and activation of a signalling cascade can result in the remodeling of the cytoskeleton; changes in morphology are visualized as changes in electrical flow (Garbison et al., 2012). Alternatively, imaging of drug-treated seeded cells has also been carried out. Typically, in these cases, immunofluorescence and fluorescence microscopy are used, which can be coupled with high-content screening techniques. Image analysis requires software to correct the image (e.g., flat field correction, background subtraction); artifact removal implies remodeling the image in such a manner as to remove autofluorescence, cell debris, and other alien fluorescent compounds.

\section{Activation of specific signaling pathways}

Binding of a ligand to its specific GPCRs leads to the activation of a specific signaling pathway and therefore ultimately to a particular set of genes. The effect of a ligand on the induction of a pathway or target genes has been measured using various methods. Reporter gene assays, cAMP measurements, and calcium-mediated signal monitoring can be used to determine whether a potential drug can activate a particular signaling cascade. In a reporter gene assay, the target gene is repla- 
ced with a reporter gene. The green fluorescent protein (GFP), $\beta$-galactosidase, firefly luciferase, Renilla luciferase, and $\beta$-lactamase are common reporter genes whose activity and levels can be easily measured (Zhang et al., 2012). However, fluorescence, similar to luminescence, can be quenched and the signal can be masked by autofluorescence of other compounds/media. The solution to this problem comes in the form of multiple alternatives: time-resolved fluorescence, fluorescence polarization, fluorescence correlation spectroscopy, and fluorescence resonance energy transfer. Among these alternatives, fluorescence polarization and fluorescence correlation spectroscopy are difficult to use with cell-based HTS (Grepin and Pernelle, 2000). Fluorescence polarization measures the rotational diffusion coefficient, while fluorescence correlation spectroscopy is based on the time required for diffusion. Thus, one measures the rotational speed and the directional speed of a diffusing molecule with the assumption that larger molecules (bound to other molecules) diffuse slower than smaller molecules. Cell-based assays, measuring diffusion of a particular molecule by bypassing the membrane, might be harder under in vitro conditions. As such, accuracy would require detection by time-resolved fluorescence or fluorescence resonance energy transfer. Further disadvantages associated with a reporter gene assay are the lengthy incubation periods, high variability, and high false positives; moreover, reporter gene assays are also the most distal target, which as noted above, can increase false positives (Thomsen et al., 2005; Michelini et al., 2010). While internal reference genes (dual reporter systems) are usually used as additional controls to circumvent these problems, these strategies require that the controls be dealt with in addition to the experimental samples, which expands sample size and increases the cost associated with the process (Michelini et al., 2010).

cAMP levels are gauged using fluorescence polarization (FP), time-resolved fluorescence (TRF), enzyme fragment complementation (EFC), time-resolved fluorescence resonance energy transfer (TR-FRET), and cAMPgated $\mathrm{Ca} 2+$ ion channel (Fang et al., 2008). Changes in calcium levels can be detected using calcium flux assays (FLIPR) or aequorin assays. The aequorin assay, for instance, utilizes a photoprotein (aequorin). The target gene is replaced with the code for apo-aequorin. Addition of coelenterazine, which binds apo-aequorin, activates aequorin; coelenterazine can traverse the mem- brane efficiently - coelenterazine is hydrophobic. Aequorin, when bound to calcium, emits luminescence (Michelini et al., 2010). Aequorin assays show high sensitivity and a high signal-to-noise ratio; however, they also display flash-type kinetics and require unique instrumentation. Neither the calcium flux assay nor the aequorin assay are applicable to inverse agonist screens; the calcium flux assay uses a normal fluorescence detection system which implies that quenching is a problem (Thomsen et al., 2005). False positives and negatives have been noted with interference (such as autofluorescence). In fact, experiments show that results vary depending on the technique used and that with the use of fluorescent techniques, a large number of compounds can be identified as leads (Gribbon and Sewing, 2003). Problems pertaining to quenching and autofluorescence were sufficiently dubitable to warrant alternate techniques. When sensitivity is required, one must resort to alternate techniques such as fluorescence polarization.

\section{Cell viability}

Cell viability is typically determined by tetrazolium or resazurin reduction, protease markers, or ATP detection, all of which measure either cell metabolism or enzymatic activity (Riss et al., 2013). The latter is done by incubating a reagent with cells to obtain a product whose concentration is assumed to be directly proportional to the number of live cells (cell death prevents product formation). MTT (permeable to the cell membrane), MTS (not permeable to the cell membrane), XTT, and WST-1 are all tetrazolium compounds that are converted by an organelle into a product that is dependent upon the concentration of tetrazolium, incubation period, cells number, and metabolic state of said cells (Riss et al., 2013). The signal obtained depends on reagent concentration, the incubation period, the number of cells, and their metabolic state; however, the incubation period is limited because the reagents are themselves toxic.

Cell viability can also be measured as a function of protease activity. GF-AFC (glycylphenylalanyl-aminofluoroumarin) is added to a cell culture where aminopeptidase converts it into AFC (fluorescent aminofluorocoumarin) (Riss et al., 2013). The advantage of this system is that it is non-toxic and therefore ideal for multiplexing, the cells are re-usable, and incubation periods are short (Riss et al., 2013). Very few studies have used 
the GF-AFC method to assess viability in high-throughput screening mode. However, Riss et al. (2013) stated that epozomicin-treated DU-145 cells subjected to the CellTiter-Fluor (GF-AFC viability assay) and the CellTiter-Glo (ATP levels) assay showed that both methods generate similar $\mathrm{EC}_{50}$ values. To assess viability, the CellTiter-Fluor (GF-AFC viability assay) was utilized amongst a number of tests with distinct purposes to isolate two potential drugs - ouabain and digoxin.

ATP assays can also be used to measure cell viability based on the consideration that non-viable cells cannot produce ATPs. In this procedure, the cells are initially lysed and the ATP content is stabilized using ATPase inhibitors. Subsequently, luciferin and luciferase are added to the medium to convert the ATP into light (Riss et al., 2013). This assay does not require incubation.

\section{Conclusions}

High-throughput screening of GPCRs using cell-based assays are typically required to assess the effect of a potential drug on a cell. In particular, one must note the possible toxic effects on cells as this is the main reason for pre-clinical and clinical rejection. A number of assays are currently available for detection of viability, changes in morphology, activation of signaling cascades, and proliferation amongst others. In particular, however, one must take note of the type of culture used (2D vs. 3D) and its optimized settings to detect valid lead compounds. Given that the detection systems are errorprone as well, selection and optimization are required to generate a valid data set.

\section{Acknowledgments}

The author would like to thank Dr. Chiara Gamberi for her collaboration.

\section{References}

Astashkina A., Mann B., Grainger D. (2012) A critical evaluation of in vitro cell culture models for high-throughput drug screening and toxicity. Pharmacol. Ther. 134: 82106.

Bedard J., May S., Barbeau D. et al. (1999) A high throughput colorimetric cell proliferation assay for the identification of human cytomegalovirus inhibitors. Antiviral Res. 41: 35-43.

Bray M.-A., Carpenter A. (2013) Advanced assay development guidelines for image-based high content screening and analysis. [in:] Assay Guidance Manual [Internet]. Ed. Sittampalam G.S., Coussens N.P., Nelson H. et al., Be- thesda (MD): Eli Lilly \& Company and the National Center for Advancing Translational Sciences. http: www.ncbi. nlm.nih.gov/books/NBK126174/.

Breier J., Radio N., Mundy W., Shafer T. (2008) Development of a high-throughput screening assay for chemical effects on proliferation and viability of immortalized human neural progenitor cells. Toxicol. Sci. 105: 119-133.

Chattopadhyay A. (2014) GPCRs: lipid-dependent membrane receptors that act as drug targets. Adv. Biol. 2014: ID 143023.

Corning Corning Microplates Product Selection Guide For Assays and Drug Discovery.

Cottet M., Faklaris O., Zwier J. et al. (2011) Original fluorescent ligand-based assays open new perspectives in G-protein coupled receptor drug screening. Pharmaceuticals 4: 202-214.

Du G., Fang Q., den Toonder J.M.J. (2016) Microfluidics for cell-based high throughput screening platforms: A review. Anal. Chim. Acta 903: 36-50.

Edmondson R., Broglie J.J., Adcock A.F., Yang L. (2014) Three-dimensional cell culture systems and their applications in drug discovery and cell-based biosensors. Assay Drug Dev. Technol. 12: 207-218.

Fang Y., Frutos A., Verklereen R. (2008) Label-free cell-based assays for GPCR screening. Comb. Chem. High Throughput Screen 11: 357-369.

Fennema E., Rivron N., Rouwkema J. et al. (2013) Spheroid culture as a tool for creating $3 D$ complex tissues. Trends Biotechnol. 31: 108-115. doi: 10.1016/j.tibtech.2012.12.003.

Fox S., Farr-Jones S., Sopchak L. et al. (2006) High-throughput screening: update on practices and success. J. Biomol. Screen 11: 864-869.

Frank An W., Tolliday N. (2010) Cell-based assays for highthroughput screening. Mol. Biotechnol. 45: 180-186.

Garbison K., Heinz B., Lajiness M. et al. (2012) Impedance-based technologies. [in:] Assay Guidance Manual [Internet]. Ed. Sittampalam G.S., Coussens N.P., Nelson H. et al., Bethesda (MD): Eli Lilly \& Company and the National Center for Advancing Translational Sciences. http: www. ncbi.nlm.nih.gov/ books/NBK91999/.

Gibbs J. (2001) Selecting the detection system - colorimetric, fluorescent, luminescent methods. ELISA Technical Bulletin.

Grepin C., Pernelle C. (2000) High-throughput screening evolution of homogeneous time resolved fluorescence (HTRF) technology for HTS. DDT 5: 212-214.

Gribbon P., Sewing A. (2003) Fluorescence readouts in HTS: no gain without pain? Drug Discov. Today 8: 1035-1043.

Johnston P., Johnston P. (2002) Cellular platforms for HTS: three case studies. Drug Discov. Today 7: 353-363.

Justice B.A., Badr N.A., Felder R.A. (2009) 3D cell culture opens new dimensions in cell-based assays. Drug Discov. Today 14: 102-107.

Kain S. (1999) Green fluorescent protein (GFP): applications in cell-based assays for drug discovery. Drug Discov. Today 4: 304-312. 
Kim L., Toh Y., Voldman J., Yu H. (2007) A practical guide to microfluidic perfusion culture of adherent mammalian cells. Lab Chip 7: 681-694.

Lodish H., Berk A., Zipursky S. (2000) Section 20.3G potein - coupled receptors and their effectors. [in:] Molecular Cell Biology. Ed. W.H. Freeman and Company, New York.

Macarron R., Banks M.N., Bojanic D. et al. (2011) Impact of high-throughput screening in biomedical research. Nat. Rev. Drug Discov. 10: 188-195.

Mcguinness R. (2007) Impedance-based cellular assay technologies: recent advances, future promise. Curr. Opin. Pharmacol. 7: 535-540.

McKim J.J. (2010) Building a tiered approach to in vitro predictive toxicity screening: a focus on assays with in vivo relevance. Comb. Chem. High Throughput Screen. 13: 188-206.

Michelini E., Cevenini L., Mezzanotte L. et al. (2010) Cell-based assays: fuelling drug discovery. Anal. Bioanal. Chem. 398: 227-222.

Papadimitriou E., Lelkes P. (1993) Measurement of cell numbers in microtiter culture plates using the fluorescent dye Hoechst 33258. J. Immunol. Meth. 162: 41-45.

Peterson K.R., Costa F.C., Fedosyuk H. et al. (2014) A cellbased high-throughput screen for novel chemical inducers of fetal hemoglobin for treatment of hemoglobinopathies. PLoS ONE 9: e107006. doi: 10.1371/journal.pone. 0107006

Riss T., Moravec R., Niles A. et al. (2013) Cell viability assays. [in:] Assay Guidance Manual [Internet]. Ed. Sittampalam G.S., Coussens N.P., Nelson H. et al., Bethesda (MD): Eli Lilly \& Company and the National Center for Advancing Translational Sciences.

Rogers M. (1997) Light on high-throughput screening: fluorescence-based assay technologies. Drug Discov. Today 2: 156-160.

Schoonen W., Westerink W., Horbach G. (2009) High-throughput screening for analysis of in vitro toxicity. [in:] Molecular, Clinical and Environmental Toxicology. Birkhäuser Basel: 401-452.
Slater K. (2001) Cytotoxicity tests for high-throughput drug discovery. Curr. Opin. Biotechnol. 12: 70-74.

Smitskamp-Wilms E., Pinedo H., Veerman G. et al. (1998) Postconfluent multilayered cell line cultures for selective screening of gemcitabine. Eur. J. Cancer 34: 921-926.

Szymański P., Markowicz M., Mikiciuk-Olasik E. (2011) Adaptation of high-throughput screening in drug discovery - toxicological screening tests. Int. J. Mol. Sci. 13: 427452. doi: 10.3390/ijms13010427.

Takakura H., Hattori M., Tanaka M., Ozawa T. (2015) Cell-based assays and animal models for GPCR drug screening. [in:] G protein-coupled receptor screening assays: methods and protocols. Humana Press, New York, Springer: 257-270.

Tautermann C.S. (2014) GPCR structures in drug design, emerging opportunities with new structures. Bioorg. Med. Chem. Lett. 24: 4073-4079.

Thomsen W., Frazer J., Unett D. (2005) Functional assays for screening GPCR targets. Curr. Opin. Biotechnol. 16: 655665.

Tung Y., Hsiao A., Allen S. et al. (2011) High-throughput 3D spheroid culture and drug testing using a 384 hanging drop array. Analyst 136: 473-478.

Yeon J., Park J. (2005) Cytotoxicity test based on electrochemical impedance measurement of HepG2 cultured in microfabricated cell chip. Anal. Biochem. 341: 308-315.

Yu H., Chung H., Yu Y. et al. (2003) A new rapid and nonradioactive assay for monitoring and determining the proliferation of retinal pigment epithelial cells. Korean J. Ophthalmol. 17: 29.

Zang R., Li D., Tang I. et al. (2012) Cell-based assays in highthroughput screening for drug discovery. Int. J. Biotechnol. Wellness Ind. 1: 31-51.

Zhang J., Chung T., Oldenburg K. (1999) A simple statistical parameter for use in evaluation and validation of high throughput screening assays. J. Biomol. Screen 4: 67-73.

Zhang Z., Guan N., Li T. et al. (2012) Quality control of cellbased high-throughput drug screening. Acta Pharm. Sin. B2: 429-438. 